\title{
Development of a STEM-EBIC/CL System
}

\author{
K.L. Bunker, R. Garcia, P.E. Russell
}

Materials Science and Engineering Department and Analytical Instrumentation Facility, North Carolina State University, 2410 Campus Shore Drive, 318 EGRC, Raleigh, NC 27695

This work involves the development and application of Scanning Electron Microscopy (SEM) and Scanning Transmission Electron Microscopy (STEM) techniques for structural, electrical and optical characterization of GaN-based heterostructures using Electron Beam Induced Current (EBIC) and Cathodoluminescence (CL). EBIC can provide information on the electrical properties of semiconductor materials and devices including electrically active defects, diffusion of carriers, and $\mathrm{p}-\mathrm{n}$ junction position. CL can provide complementary information including radiative recombination and luminescence homogeneity.

Previously, SEM-based EBIC was performed in a Hitachi S-3200N SEM to obtain the minority carrier diffusion length in a GaN-based heterostructure [1]. In addition, a STEM-EBIC system was designed and implemented in a Hitachi HD-2000 STEM in order to characterize an InGaN quantum well heterostructure. The relative position of the $p-n$ junction with respect to a thin InGaN quantum well was resolved with the STEM-EIBC technique with nanometer precision [2].

In this work, an improved holder has been designed specifically for simultaneous EBIC and CL experiments and a sample preparation technique has been developed for fully packaged devices. In STEM-EBIC experiments, the sample must be electrically active and the region of interest must be electron transparent. The samples chosen to demonstrate the new sample preparation technique were commercially available AlGaInP MQW devices (Fig. 1 (a)). The sample was embedded in glue and mechanically polished into a wedge. The wedge was placed between two $3 \mathrm{~mm}$ half $\mathrm{Cu}$ grids, each grid making contact to one side of the device. Focused Ion Beam (FIB) milling was used to create an electron transparent membrane of $\sim 100 \mathrm{~nm}$ in the active region of the device (Fig. 1 (b)). The electrical activity of the device is checked by applying a bias across the two $\mathrm{Cu}$ grids (Fig. 1 (c)).

The custom sample holder was designed with an insulating piece of Macor ${ }^{\circledR}$ seated in the tip of the specimen rod (Fig. 2 (a)). The electrically active device is placed on a piece of gold that is located in the $3 \mathrm{~mm}$ cup of the specimen holder, making contact to one of the $\mathrm{Cu}$ grids. A Cu-Be clip holds the sample in place and makes contact to the other $\mathrm{Cu}$ grid. Two isolated electrical feedthroughs make contact via a screw to the gold piece and the $\mathrm{Cu}$-Be clip.

The implementation of a STEM-CL system in a Hitachi HD-2000 STEM allows for the simultaneous structural, chemical, electrical and optical properties of semiconductors and devices. The space limitations imposed by the pole pieces and column port make the design of a CL system challenging (Fig. 2 (b) and (c)). Several different detector designs are being investigated including the implementation of commercial photodetector. Preliminary SEM-CL experiments using a modified $5.1 \mathrm{~mm}^{2}$ silicon photodetector have been performed on a GaN-based heterostructure in a Hitachi S-3200N. The simultaneous collection of EBIC and panchromatic CL images was demonstrated and a mean EBIC and CL linescan was obtained (Fig. 3 (a) and (b)).

In addition, the new STEM-EBIC/CL holder will make it possible to positively and negatively bias the device in the Hitachi HD-2000 STEM and observe any effects in the electrical and optical properties of the device. The effects of biasing the device on the EBIC and CL linescans is currently being investigated in a JEOL 6400 Field Emission SEM and a Hitachi S-3200N SEM. 
[1] J.C. Gonzalez et al., Applied Physics Letters, 79 (2001) 1567.

[2] K.L. Bunker et al., Electronic Device Failure Analysis, 4 (2002) 29.
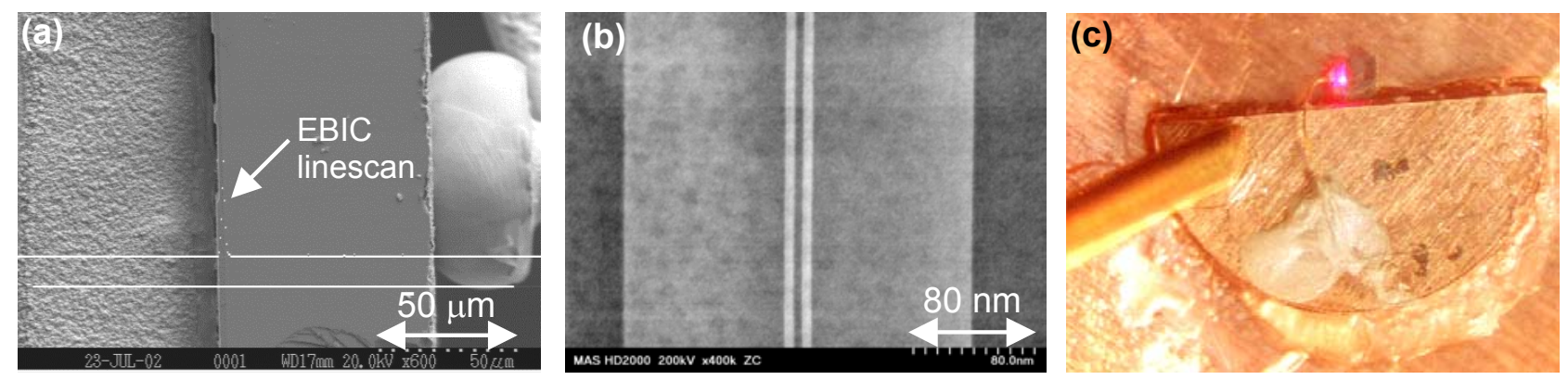

Fig.1 (a) EBIC signal overlaid on $20 \mathrm{keV}$ Secondary Electron (SE) image showing the location of electrical junction approximately 2 microns from the bottom of the device taken in a Hitachi S-3200N SEM. (b) Z-Contrast image of quantum well structure after FIB milling taken in a Hitachi HD-2000 STEM. (c) Fully prepared sample emitting under bias.

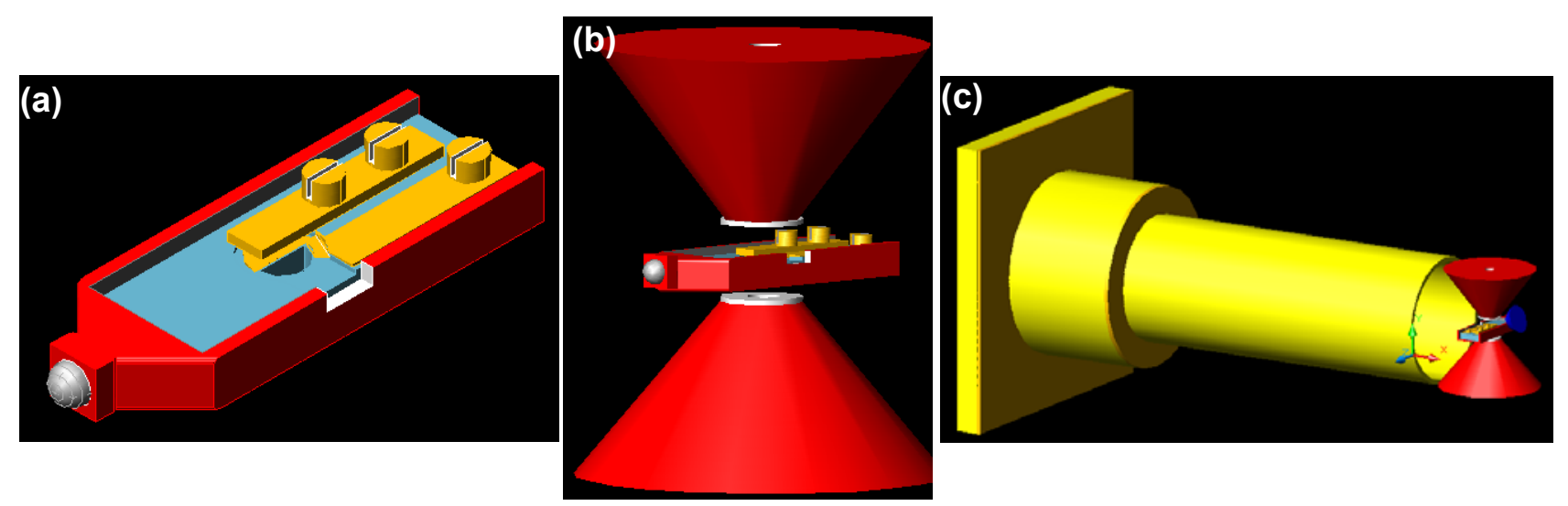

Fig.2 (a) Fully assembled STEM-EBIC/CL holder. (b) Sample holder placed between the two pole pieces of the Hitachi HD-2000 STEM. (c) Open port available for CL detector on HD-2000 STEM.
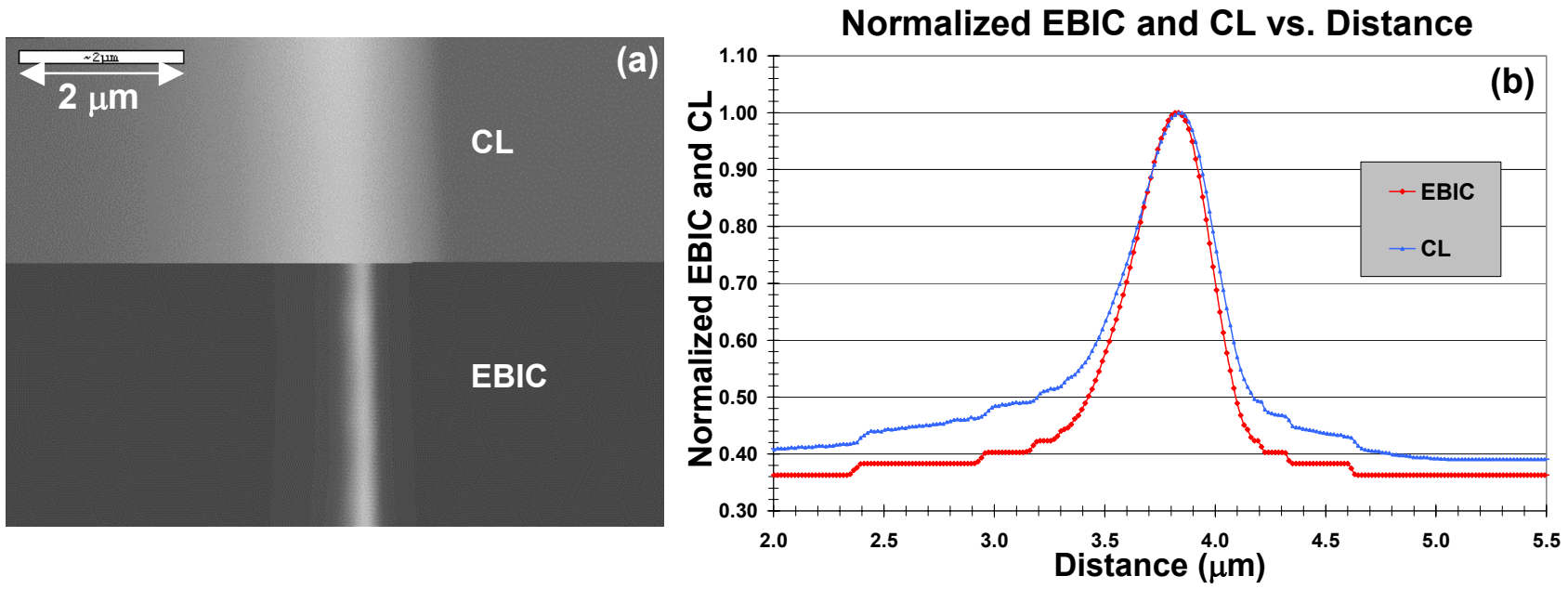

Fig.3 (a) SEM-EBIC and panchromatic CL images collected at $30 \mathrm{keV}$ and a beam current of $4 \mathrm{nA}$ in a Hitachi S$3200 \mathrm{~N}$ SEM. (b) Average EBIC and CL linescans obtained by averaging 100 lines in the EBIC and CL images. 